\title{
Schäfer, Sebastian: Rudolf Olden - Journalist und Pazifist. Vom Unpolitischen zum Pan-Europäer. Moralische Erneuerung im Zeichen moderner Kulturkritik (Weimarer Schriften zur Republik, Bd. 8), 438 S., Steiner, Stuttgart 2019.
}

\author{
Annika Klein
}

Angenommen: 4. Dezember 2020 / Online publiziert: 30. Dezember 2020

(C) Der/die Autor(en) 2020

Wer sich mit der politischen Geschichte der Weimarer Republik auseinandersetzt, dem ist Rudolf Olden zumindest als Rechtsanwalt Carl von Ossietzkys im „Weltbühne“-Prozess bekannt, der für diesen einen Freispruch erwirken konnte. Olden hatte sich in der Zwischenkriegszeit aber vor allem als Journalist einen Namen gemacht, der als kritischer Beobachter die politischen Entwicklungen in Deutschland zunächst von Wien aus kommentierte, dann für das „Berliner Tageblatt“ direkt aus der Hauptstadt berichtete.

Sebastian Schäfer hat sich zum Ziel gesetzt, in seiner Biografie insbesondere den Pazifisten Olden in den Blick zu nehmen und dabei Ideengeschichte und Intellektuellengeschichte zu einer, ,intellektuellen Biographie“ zu verbinden, um so die zwangsläufig der Biografie innewohnende Selektivität produktiv zur Analyse des Pazifismus Oldens zu nutzen. Ein zweiter Schwerpunkt liegt auf der Frage, ob Olden in seinen verschiedenen Rollen, als Schriftsteller und Journalist, als Jurist und Rechtsanwalt und eben als Pazifist auch der Gruppe der Intellektuellen zugerechnet werden kann.

Dem eigentlichen biografischen Teil stellt Schäfer einen Überblick über die verschiedenen Strömungen und Gruppierungen der Friedensbewegung in Deutschland voran, die sich seit Ausbruch des Ersten Weltkrieges beziehungsweise als Reaktion darauf entwickelt hatten. Es folgen in Kapitel drei kurze Schlaglichter auf den familiären Hintergrund und die Jugend Oldens sowie die ersten Kontakte zur Wiener Moderne und eine mögliche Zukunft als Schriftsteller. Zur Friedensbewegung positionierte sich Olden in dieser Zeit nicht, auch wenn Schäfer mit Jakob Wassermann ein mögliches pazifistisches Vorbild auszumachen glaubt.

\footnotetext{
A. Klein $(\bowtie)$

Johann Wolfgang Goethe-Universität Frankfurt a. M., Frankfurt a. M., Deutschland

E-Mail: A.Klein@em.uni-frankfurt.de
} 
Kapitel vier widmet sich dem Ersten Weltkrieg und Oldens „Politisierung im Schützengraben“, wo er Phasen der Kriegsbegeisterung, der Langeweile und schließlich Ernüchterung durchlief. Eine kurzzeitige Versetzung an ein Kriegsgericht führte offenbar zu einer endgültigen Desillusionierung, wenngleich Schäfer selbst eingesteht, dass sich kaum konkretere Informationen zu Oldens politischer - oder pazifistischer - Einstellung finden lassen. In den „Wiener Jahren“ der frühen Nachkriegszeit (Kapitel fünf) positionierte er sich als Journalist und Herausgeber eindeutiger zu Republik, Demokratie, rechtsstaatlichen Lösungen und der Pan-Europa-Idee als Grundlagen inneren und äußeren Friedens, vereinte dabei aber Standpunkte des gemäßigten und des radikalen Pazifismus und vermied es, sich dezidiert einer der pazifistischen Gruppierungen in Deutschland anzuschließen.

Mitte der 1920er Jahre wechselte Olden in die Redaktion des „Berliner Tageblatt“; auf die Wiener folgten die „Berliner Jahre“ (Kap. 6). Für das „Tageblatt“ schrieb er vor allem über die Krise der Justiz, die Aktivitäten der Schwarzen Reichswehr und die Femeprozesse. Gleichzeitig partizipierte er als Rechtsberater der „Republikanischen Beschwerdestelle“ nicht nur aktiv am Justizwesen, sondern in der ,Deutschen Liga für Menschenrechte“ zudem erstmals in einer pazifistischen Organisation. Für die Beschwerdestelle betreute Olden zahlreiche politische Prozesse, von denen die Verteidigung Carl von Ossietzkys im „Weltbühne“-Prozess wohl die weitreichendste Beachtung fand. Diese eindeutige politische Positionierung war es aber scheinbar auch, die angesichts einer konträren Bewegung von Presse und Politik Ende 1931 zu Oldens Entlassung aus der Redaktion des „,Tageblattes“ führte.

Nach dem Reichstagsbrand entging Olden nur knapp der Verhaftung und floh nach London. Im „Exil““ (Kapitel sieben) stellte er sich klar gegen die, in seinen Augen, zu passive und zu pazifistische Haltung der britischen Politik gegenüber den Nationalsozialisten und bemühte sich in Artikeln und Monografien - wenig erfolgreich - um die „Aufklärung“ der britischen Öffentlichkeit. Zudem scheiterte sein Versuch, die britische Staatsangehörigkeit zu erlangen. Nach kurzzeitiger Internierung gestattete man ihm jedoch die Ausreise in die USA, die er nicht mehr erreichte: im August 1940 wurde das Schiff, auf dem sich Olden und seine Frau befanden, von einem deutschen U-Boot versenkt.

Schäfers Versuch, an Olden als einen „scheinbar Vergessenen“ (S. 7) zu erinnern und anstelle einer klassischen Biografie das Modell der intellektuellen Biografie beziehungsweise einer ,personalisierten Ideengeschichte“ (S. 17) zur Ausleuchtung der verschiedenen Facetten Oldens und seiner oft von den Hauptströmungen des Pazifismus abweichenden Positionen zu präsentieren, ist streckenweise gut gelungen. Der Autor kann interessante Bezüge zwischen den verschiedenen Rollen Oldens als Schriftsteller, als journalistischer Beobachter aus der Wiener Distanz und schließlich als sowohl journalistisch wie auch juristisch aktiv am politischen Geschehen Beteiligter vor dem Hintergrund größerer ideengeschichtlicher und politischer Entwicklungen herstellen. Er ist erkennbar bemüht, den oft durch das Quellenmaterial schwer greifbaren Einflüssen auf Oldens politische und pazifistische Prägung im Detail nachzuspüren und dabei Grad und Wahrscheinlichkeit der tatsächlichen Beeinflussung kritisch zu hinterfragen.

Genau darin liegt aber leider auch eine Schwäche des Buches: Die Versuche, Olden unbedingt einer spezifischen Ausprägung des Pazifismus der Zwischenkriegszeit 
oder einem Konzept des Intellektuellen zuordnen zu wollen, wirken häufig sehr konstruiert, gerade weil der Verfasser selbst eingestehen muss, dass sich Olden entweder nicht zu spezifischen Punkten äußerte, oder aber Positionen vertrat und Rollen verkörperte, die im Rahmen dieser Zuordnungsschemata widersprüchlich oder unpassend erscheinen. Auch von einer strukturellen und sprachlichen Überarbeitung hätte das Buch profitiert, fehlende Überleitungen und abrupt einsetzende lange Exkurse lassen einen roten Faden zuweilen schwer erkennen; dazu kommt eine Vielzahl von Grammatikfehlern. Das ist bedauerlich, denn Schäfers Buch eröffnet durchaus eine Vielzahl neuer Perspektiven auf Rudolf Olden, an die sich hoffentlich anknüpfen lässt.

Funding Open Access funding enabled and organized by Projekt DEAL.

Open Access Dieser Artikel wird unter der Creative Commons Namensnennung 4.0 International Lizenz veröffentlicht, welche die Nutzung, Vervielfältigung, Bearbeitung, Verbreitung und Wiedergabe in jeglichem Medium und Format erlaubt, sofern Sie den/die ursprünglichen Autor(en) und die Quelle ordnungsgemäß nennen, einen Link zur Creative Commons Lizenz beifügen und angeben, ob Änderungen vorgenommen wurden.

Die in diesem Artikel enthaltenen Bilder und sonstiges Drittmaterial unterliegen ebenfalls der genannten Creative Commons Lizenz, sofern sich aus der Abbildungslegende nichts anderes ergibt. Sofern das betreffende Material nicht unter der genannten Creative Commons Lizenz steht und die betreffende Handlung nicht nach gesetzlichen Vorschriften erlaubt ist, ist für die oben aufgeführten Weiterverwendungen des Materials die Einwilligung des jeweiligen Rechteinhabers einzuholen.

Weitere Details zur Lizenz entnehmen Sie bitte der Lizenzinformation auf http://creativecommons.org/ licenses/by/4.0/deed.de. 